\title{
Evaluierung von Benutzeranforderungen für die Kommunikation zwischen automatisierten Fahrzeugen und ungeschützten Verkehrsteilnehmern
}

\author{
Philipp Wintersberger, Andreas Löcken, Anna-Katharina Frison \\ und Andreas Riener
}

Es wurden bereits einige Lösungen vorgestellt, um die Kommunikation zwischen automatisierten Fahrzeugen und ungeschützten Verkehrsteilnehmern (sog. „Vulnerable Road Users“, VRUs) zu unterstützen. Noch ist jedoch unklar, ob diese Systeme den Anforderungen zukünftiger Benutzer auch gerecht werden. Ziel dieser Arbeit war es, Benutzeranforderungen von VRUs nach direktem Kontakt mit einem automatisierten Fahrzeug zu erfassen. Hierfür wurde eine Feldstudie mit 32 Teilnehmern durchgeführt. Die Resultate, welche sowohl auf subjektiven (Fragebögen, Interviews) als auch objektiven (Videoanalyse) Daten basieren, legen nahe, dass ungeschützte Verkehrsteilnehmer einfache und bekannte Konzepte (beispielsweise Ampelsysteme oder Hupen) zur Kommunikation bevorzugen. Des Weiteren wurden diverse Problemszenarien identifiziert, welche für eine Bereitstellung von automatisierten Fahrzeugen in „Shared Spaces“ von besonderer Bedeutung sind.

P. Wintersberger · A. Löcken

Technische Hochschule Ingolstadt, Forschungszentrum CARISSMA, Ingolstadt, Deutschland E-Mail: philipp.wintersberger@carissma.eu; andreas.loecken@carissma.eu

A.-K. Frison · A. Riener $(\bowtie)$

Technische Hochschule Ingolstadt, Ingolstadt, Deutschland

E-Mail: anna-katharina.frison@thi.de; andreas.riener@thi.de 


\subsection{Einleitung}

Spätestens seit erste automatisierte Fahrzeuge im Realverkehr getestet werden, tritt eine neue Frage immer mehr in den Vordergrund: Wie können diese mit anderen Verkehrsteilnehmern kommunizieren, wenn kein Insasse mehr als Fahrer permanent am Geschehen teilnimmt (Mahadevan et al. 2018; Mirnig et al. 2018)? Menschliche Verkehrsteilnehmer nutzen üblicherweise formlose Kommunikation, um Konflikte, z. B. Entscheidung über Vorrang in nicht-geregelten Situationen, zu vermeiden beziehungsweise aufzulösen. Dies umfasst nicht nur explizite Kommunikation (etwa Blinken, Hupen oder das Suchen von Augenkontakt), sondern auch Fahrverhalten oder Bewegungsmuster (Färber 2015). Welche Verhaltensmuster hierbei ausschlaggebend sind, ist nicht direkt geregelt, trotzdem schaffen es Menschen üblicherweise, die Signale anderer korrekt zu interpretieren und dabei Konfliktsituationen, meist unbewusst, zu lösen. Um diese Art der Kommunikation in automatisierten Fahrzeugen zu ersetzen, wurden sowohl von Fahrzeugherstellern als auch Forschern bereits verschiedenste Lösungen vorgeschlagen, wie etwa in den Außenraum gerichtete Bildschirme, die situationsabhängige Projektion von Fußgängerüberwegen, Visualisierungen mit Licht- und Tonsignalen bis hin zu geräteübergreifenden Systemen mit Smartphones, Smartwatches oder anderen ,Wearable Devices“ (Dey et al. 2018; Mahadevan et al. 2018; Mirnig et al. 2018). Dabei ist jedoch anzumerken, dass es nicht reichen wird, „nur“ technisch ausgefeilte Konzepte zu präsentieren. Um die Verkehrssicherheit beim Mischbetrieb mit automatisierten Fahrzeugen zu erhalten oder gar zu erhöhen, müssen derartige Systeme neuen, zu definierenden Standards folgen. Dabei könnte eine Koexistenz verschiedenster Lösungen potenziell für Verwirrung sorgen - ein Problem, welches gerade für ungeschützte Verkehrsteilnehmer/VRUs (wie etwa Fußgänger, Rad- oder Rollstuhlfahrer) kritisch werden könnte, da diese laut Statistiken einen immer größeren Teil der bei Unfällen verunglückten Personen ausmachen (OECD 2014).

Bevor man somit beginnt, technisch ausgefeilte Lösungen zu entwerfen, ist es notwendig, die Erwartungen von VRUs zu erfassen, und deren spezielle Anforderungen beim Systemdesign zu berücksichtigen. Unter diesem Gesichtspunkt können reale Interaktionen zwischen automatisierten Fahrzeugen und VRUs eine äußerst wertvolle Basis für weitere Forschung und potenzielle Systementwürfe bieten. Um Anforderungen von Fußgängern an derartige Kommunikationssysteme zu erheben, wurde eine Feldstudie mit einem im Realverkehr operierenden automatisierten Shuttle durchgeführt. Ziel der Studie war es, durch die Zusammenführung von Fragebögen, Interviews und Videobeobachtungen potenzielle Probleme und Anforderungen für derartige Kommunikationssysteme zu identifizieren. Dabei wurde die persönliche Sichtweise nur von jenen Personen erfasst, die unmittelbar zuvor als ungeschützte Verkehrsteilnehmer Kontakt mit einem automatisierten Shuttle hatten. Die Ergebnisse der Befragungen in Kombination mit den aus der Videobeobachtung erhaltenen Daten sollen helfen, Kommunikationssysteme zukünftiger Fahrzeuge einfacher und auch sicherer zu gestalten. 


\subsection{Stand von Wissenschaft und Technik}

VRUs nutzen eine Reihe von Informationen, um Entscheidungen im Straßenverkehr (z. B. das Überqueren einer Straße) zu treffen, wie etwa Entfernung, Geschwindigkeit, Bremsund Beschleunigungsverhalten anderer Verkehrsteilnehmer, aber auch Sichtbedingungen und Sicherheitsabstände (Sucha et al. 2017). Wie Fußgänger sich beim Überqueren einer Straße verhalten, wurde bereits in Studien, hauptsächlich im Kontext des manuellen Fahrens, untersucht, und zwar sowohl in simulierter (Lehsing et al. 2016) als auch realer (Jiang et al. 2015; Jain et al. 2014) Umgebung. Einige Studien bescheinigen dem Suchen von Augenkontakt eine besondere Relevanz (Sucha 2014; Lundgren et al. 2017), andere fügten hinzu, dass explizite Kommunikation zumeist nur dann nötig wird, wenn sich Verkehrsteilnehmer auffällig verhalten (Dey und Terken 2017; Rothenbücher et al. 2016). Solange das Verhalten der einzelnen Akteure vorhersehbar ist, passen diese sich durch Vorhersagen der Trajektorien gegenseitig an. Jedoch wird bei automatisierten Fahrzeugen darauf hingewiesen, dass Verkehrsteilnehmer zusätzlich mit explizit kommunizierenden Systemen angesprochen werden sollten (Rothenbücher et al. 2016) Derartige, zumeist an Fußgänger gerichtete Systeme, wurden schon von einigen Fahrzeugherstellern vorgestellt (siehe Abb. 7.1). Der F 015 von Mercedes ${ }^{1}$ projiziert einen Fußgängerüberweg auf die Straße, beim Nissan IDS $^{2}$ wurden mehrfarbige LED-Lampen an den Fahrzeugseiten angebracht. Der I.D. BUZZ Microbus ${ }^{3}$ von VW versucht via LEDs an der Front Augen nach-
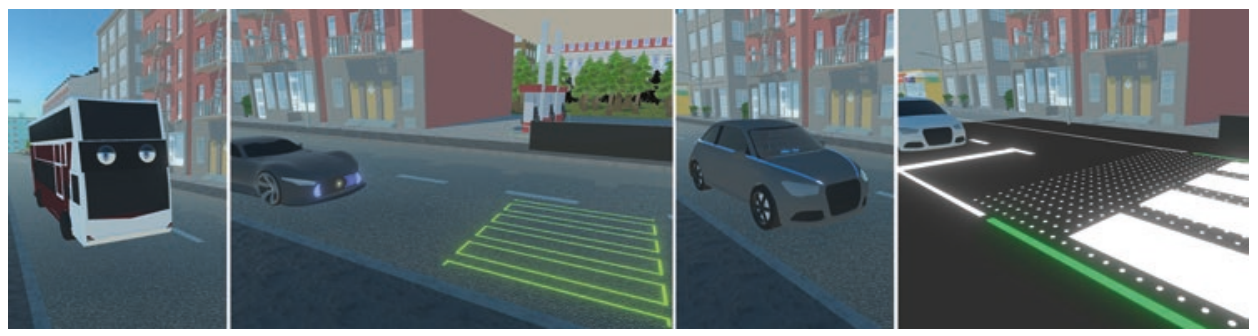

Abb. 7.1 Darstellung bereits vorgestellter Konzepte für AV-VRU-Kommunikation (von links nach rechts): Anblicken/Nachverfolgen eines VRUs mit künstlichen Augen, Projektion eines Fußgängerüberwegs, LED-Streifen und Displays, bzw. „Smart Infrastructure“ (Fußgängerübergang in der Straße integriert). (Quelle: eigene Darstellung)

\footnotetext{
${ }^{1}$ https://www.theverge.com/2015/3/20/8263561/mercedes-benz-f-015-self-driving-video. Zugegriffen am 25.01.2019.

${ }^{2}$ https://www.forbes.com/sites/matthewdepaula/2015/10/28/nissan-ids-concept-showcases-the-future-of-autonomous-driving/. Zugegriffen am 25.01.2019.

${ }^{3}$ https://insideevs.com/volkswagen-id-buzz-iconic-microbus-reborn-270-electric-vehicle/. Zugegriffen am 25.01.2019.
} 
zubilden, andere Konzepte setzen auf Displays, wie etwa Drive.ai ${ }^{4}$ auf der Oberseite des Fahrzeuges, oder wie in einem Patent von Google beschrieben an der Fahrzeugfront sowie den Seiten. Auch von akademischer Seite wurden bereits mehrere Konzepte präsentiert. Eines der ersten war AEVITA vom Massachusetts Institute of Technology, welches jedoch weniger einem klassischen Fahrzeug, denn einem fahrenden Roboter entsprach. Dieser versuchte, über gerichteten Schall sowie Lampen bzw. farbwechselnde Räder menschliches Verhalten nachzubilden (Pennycooke 2012). Im Projekt AVIP wurden mehrere Lösungen, z. B. LED-Matrizen auf dem Kühlergrill, über der Windschutzscheibe, aber auch Laserprojektionen vorgestellt (Lagstrom und Lundgren 2015). Ein von Böckle et al. (2017) vorgestelltes, und in virtueller Realität evaluiertes, Projekt nutzte auch verschiedene Lichter an der Frontpartie eines automatisierten Shuttles. Mahadevan et al. (2018) verglichen vier prototypische Interfaces sowohl mit einem automatisierten Fahrzeug als auch mit einem Segway und hoben hervor, dass zukünftige Kommunikationssysteme mehrere Modalitäten gleichzeitig unterstützten sollten. So vielfältig die Konzepte auch sind, ein direkter Vergleich fällt schwer oder ist unmöglich. Wissenschaftliche Prototypen nutzen verschiedenste Methoden zur Evaluierung, bei Industrieprojekten werden oft keine Evaluationen durchgeführt bzw., falls doch, die Ergebnisse nicht veröffentlicht.

Andererseits muss jedoch die Frage gestellt werden, ob es überhaupt sinnvoll ist, eine hohe Anzahl teils komplett unterschiedlicher Konzepte einzusetzen bzw. zu genehmigen. Viele der vorgestellten Lösungen wurden zudem in nur wenigen Szenarien (und primär mit subjektiven Evaluierungsmethoden) getestet. Um für zukünftigen Einsatz gerüstet zu sein, sollen derartige Kommunikationssysteme jedoch verschiedenste Kriterien erfüllen (Mirnig et al. 2018): Sie müssen (in zu definierenden standardisierten Testverfahren) validierbar sein, kulturelle und auch individuelle Unterschiede berücksichtigen, in vielen verschiedenen Umgebungen (z. B. bei unterschiedlichen Sichtverhältnissen) funktionieren, dabei möglichst skalierbar (d. h. auch in komplexen Situationen mit mehreren Fahrzeugen und VRUs) arbeiten können. Die Zielerreichung ist dabei nicht zwingend widerspruchsfrei möglich - zum Beispiel wird darauf hingewiesen, dass Fußgänger Entscheidungen aufgrund von Bewegungsmustern (z. B. Abbremsen) treffen (Dey und Terken 2017), was sich jedoch negativ auf den Verkehrsfluss oder den Energieverbrauch auswirken kann. Zusätzlich zu den hier genannten Aspekten sollen diese Systeme auch den Erwartungen potenzieller Nutzer entsprechen und auf in der Realität vorkommende Probleme zugeschnitten sein - beides Punkte, welche im Rahmen der hier vorgestellten Studie untersucht wurden.

\subsection{Feldstudie}

Um die Anforderungen von VRUs besser zu verstehen, wurde eine Feldstudie mit einem im Realverkehr operierenden automatisierten Shuttle (EasyMile EZ10) durchgeführt.

\footnotetext{
${ }^{4}$ https://www.drive.ai/. Zugegriffen am 25.01.2019.
} 


\subsubsection{Methode und Forschungsfragen}

Durch Triangulation verschiedener Forschungsmethoden sollen sowohl die subjektiven Erwartungen von Benutzern als auch sich durch das Zusammentreffen des Shuttles mit VRUs ergebende Probleme erhoben werden. Dabei wurden Personen, welche unmittelbar davor dem Shuttle begegnet waren, in semi-strukturierten Interviews befragt und ihre generelle Einstellung gegenüber automatisierten Fahrzeugen in Form eines kurzen Fragebogens erfasst. Dieser basiert auf der Studie von Reig et al.(2018), welche in einem ähnlichen Setting automatisierte Taxis von Uber evaluierte. Ein Ergebnis dieser Studie war, dass ein direkter Zusammenhang zwischen der Einstellung gegenüber automatisierten Fahrzeugen und künstlicher Intelligenz beruht. In der hier präsentierten Studie wurden zusätzlich noch weitere Fragen über allgemeine Technikakzeptanz sowie potenzielle Kommunikationsprobleme aufgenommen. Des Weiteren wurde die Kurzversion des AttrakDiff-Fragebogens (Hassenzahl et al. 2003) hinzugefügt. Dieser erfasst die für das Benutzererlebnis relevanten Konzepte Attraktivität, sowie pragmatische (Effektivität und Effizienz eines Produktes) und hedonische (über reine Nützlichkeit hinausgehende Produkteigenschaften, wie Identifikation, Stimulation, etc.) Qualität auf einer 5-Punkte Likert-Skala von -2 bis +2 . Auch die Interviewfragen selbst wurden an die Studie von Reig et al. (2018) angelehnt und um zusätzliche Fragen ergänzt (siehe Anhang 2). Dadurch sollte eine Vergleichbarkeit zwischen der Studie von Reig et al. und unserer Evaluation gegeben sein. Für die Auswertung der Interviews wurde eine Inhaltsanalyse mit ,Affinity Diagramming“ (Courage und Baxter 2005) der Antworten durchgeführt. Dabei wurden häufig vorkommende Statements geclustert und bestimmten wiederkehrenden Themen zugeordnet.

Um nicht nur das subjektive Empfinden von VRUs zu evaluieren, sondern auch objektiv existierende Probleme im Umgang mit dem automatisierten Shuttle zu erfassen, wurde zusätzlich eine Videoanalyse durchgeführt. An zwei aufeinander folgenden Tagen (November 2018) wurde eine Kamera am Shuttle befestigt und damit Videoaufnahmen des Außenraumes aufgezeichnet. In einer manuellen Videoanalyse wurden potenziell problematische Situationen annotiert und anschließend, ähnlich wie bei der Analyse der Interviewdaten, in zusammenpassende Themenbereiche gegliedert. Durch die unterschiedliche Annäherung an das Thema sollen folgende Forschungsfragen beantwortet werden:

- RQ1 Welche Anforderungen haben VRUs an die Kommunikation mit automatisierten Fahrzeugen und welche Lösungsansätze werden vorgeschlagen?

- RQ2 Welche Situationen führen bei der Interaktion zwischen VRUs und automatisierten Fahrzeugen zu Problemen?

\subsubsection{Studienablauf}

Für die Fragebögen und Interviews wurden speziell Personen gesucht, welche unlängst mit dem automatisierten Shuttle interagiert hatten. Hierfür platzierte sich unser Ver- 
suchspersonal an markanten Punkten entlang der Strecke (z. B. Vorrangstraße, Fußgängerübergang, etc.) und sprachen Personen nach deren Zusammentreffen mit dem Shuttle an. Es wurde darauf geachtet, sowohl ältere (Kurgäste, Anwohner), als auch jüngere Personen (z. B. Schüler einer nahegelegenen Akademie) in die Evaluation aufzunehmen. Personen, welche sich zur Studienteilnahme bereit erklärten, füllten zuerst den Fragebogen mit demografischen Daten, den Fragen zu Akzeptanz und Kommunikationsproblemen, sowie den AttrakDiff Fragebogen aus. Anschließend wurde das semi-strukturierte Interview geführt und aufgezeichnet. Die Durchführung dauerte in etwa 20 Minuten pro Teilnehmer.

\subsubsection{Resultate}

Insgesamt nahmen 32 (19 männliche, 13 weibliche) Probanden im Alter von 17 bis 89 Jahren an der Studie teil. Es wurden speziell ältere und jüngere Probanden gesucht, um gegebenenfalls Unterschiede in den Altersgruppen zu identifizieren. Die Gruppe der jüngeren Probanden umfasste 17 Personen (M: 21, SD: 4,27 Jahre), darunter zwei, die keine genauen Angaben zu ihrem Alter machten, deren Zuteilung zu dieser Gruppe jedoch aufgrund des Aussehens möglich war, die Gruppe der älteren Probanden 15 Personen (M: 71,7, SD: 9,78 Jahre). Nachfolgend werden die Ergebnisse der Fragebögen, Interviews und der Videoanalyse präsentiert.

\subsubsection{Fragebögen}

Technikakzeptanz und Einstellung gegenüber automatisierten Fahrzeugen

Dieser Teil des Fragebogens umfasste 19 Items, wobei zwölf (jeweils vier) die Akzeptanz gegenüber automatisierten Fahrzeugen (AAV), künstlicher Intelligenz (AKI), sowie technischen Neuerungen (AT) allgemein, sowie zwei weitere Items die Vorhersehbarkeit (PRED) des Verhaltens des automatisierten Shuttles adressierten. Mit den restlichen fünf Fragen wurde das Verhalten (BEHAVIOR), die kognitive Belastung (MENTAL), die Risikobereitschaft (RISK), der Wunsch zur spielerischen Auseinandersetzung (PLAY) sowie die Schwierigkeit der Kommunikation (COMM) bei Interaktionen mit dem Shuttle erfasst (siehe Anhang 1). Bei Betrachtung der Mediane (siehe Tab. 7.1) fällt auf, dass sämtliche Konzepte von den Teilnehmern positiv bewertet wurden.

Da die jeweilig zusammengehörigen Items akzeptable Werte für die interne Reliabilität aufwiesen (Cronbach's alpha: $A A V=0,80, A K I=0,92, A T=0,87, P R E D=0,81$ ) wurden Skalenvariablen (Mittelwerte) für die weitere Analyse gebildet. Anschließend wurde bezüglich der verschiedenen erfassten Konzepte eine Korrelationsanalyse (Spearman) durchgeführt. Diese zeigt, dass die jeweiligen Subskalen für Akzeptanz stark miteinander korrelieren (AAV/AKI: $r=0,640, p<0,001$, AAV/AT: $r=0,473, p=0,004$, AKI/AT: $r=0,554, p=0,004$ ). Dies bestätigt die Resultate von (Reig et al. 2018) und zeigt zusätzlich: Eine hohe Akzeptanz automatisierter Fahrzeuge geht einher mit Akzeptanz gegenüber künstlicher Intelligenz und technischen Neuerungen im Allgemeinen. 
Tab 7.1 Deskriptive Statistik der im Fragebogen erhobenen Konzepte

\begin{tabular}{l|l|l|l}
\hline Konzept & Inhalt & Mdn & IQR \\
\hline AAV & Akzeptanz automatisierter Fahrzeuge & 0,5 & 1,31 \\
\hline AKI & Akzeptanz gegenüber künstlicher Intelligenz & 0,63 & 1,31 \\
\hline AT & Akzeptanz gegenüber neuen Technologien & 1 & 1,06 \\
\hline PRED & Vorhersehbarkeit von Aktionen des automatisierten Fahrzeuges & 0,5 & 1,1 \\
\hline BEHV & Verhaltensänderung gegenüber manuellen Fahrzeugen & 1 & 1 \\
\hline RISK & Risikobereitschaft beim Queren der Straße & 1 & 3 \\
\hline PLAY & Wunsch nach spielerischer Auseinandersetzung & 1 & 3 \\
\hline COMM & Kommunikationsbedarf & 1 & 2 \\
\hline
\end{tabular}

Zusätzlich korreliert AAV negativ mit COMM $(r=-0,533, p=0,001)$. Personen, welche somit automatisierte Fahrzeuge akzeptieren, verneinen, dass es schwierig ist, mit diesen zu kommunizieren. Zwei weitere Korrelationen betreffen RISK. Hier wurde abgefragt, ob man einem automatisierten Fahrzeug aufgrund fehlender Kommunikationsmöglichkeiten generell die Vorfahrt gibt. RISK korreliert dabei negativ mit PRED ( $r=-0,458, p$ $=0,005)$ und positiv mit MENTAL $(r=-0,357, p=0,026)$. Personen, welche glauben, das Verhalten des Fahrzeugs vorhersagen zu können, haben somit keine Scheu vor diesem die Straße zu überqueren (und umgekehrt), während Probanden, welche glauben, im Umgang mit automatisierten Fahrzeugen überfordert zu sein, dies nicht riskieren. Statistische Vergleiche zwischen den jeweiligen Subskalen zeigen weder in Punkto Geschlecht noch Altersgruppe besondere Unterschiede - einzig, was die Akzeptanz betrifft, lieferte der durchgeführte Mann-Whitney U Test ein statistisch signifikantes Ergebnis ( $p=$ 0,017), wobei ältere Probanden eine höhere Akzeptanz gegenüber dem Fahrzeug aufwiesen.

\section{AttrakDiff}

Im Schnitt wurden sämtliche Produkteigenschaften von den Probanden positiv bewertet. Wie in Abb. 7.2 ersichtlich, beträgt der Median für Attraktivität in beiden Altersgruppen $0,5\left(\mathrm{IQR}_{\text {jung }}: 1,375, \mathrm{IQR}_{\text {alt }}: 1\right)$, wodurch sich keine statistischen Unterschiede zwischen den Gruppen ergeben. Auch bei der hedonischen Qualität konnten keine Unterschiede zwischen den Gruppen gefunden werden $\left(\mathrm{Mdn}_{\text {jung }}: 0,5, \mathrm{IQR}_{\text {jung }}: 0,5, \mathrm{Mdn}_{\text {alt }}: 0,75 \mathrm{IQR}_{\text {alt }}: 0,75\right)$. Pragmatische Qualität hingegen wurde von jüngeren Versuchspersonen (Mdn: 0,375, IQR: 1,375) um einiges niedriger bewertet als von älteren Teilnehmern (Mdn: 1, IQR: 1,5); ein, Effekt der sich nach Durchführung eines Mann-Whitney-U-Tests als statistisch signifikant erwies $(U=44,5, Z=-2,27, p<0,05, r=0,44)$.

\subsubsection{Interviews}

Die Aufnahmen der aus den direkt im Anschluss an die Fragebögen (siehe Anhang 7.7.2) geführten Interviews wurden transkribiert und in einzelne Statements unterteilt. Diese wurden anschließend mit „Affinity Diagramming“ (Courage und Baxter 2005) einer Inhaltsanalyse unterzogen und in wiederkehrende Themenbereiche gegliedert. Im Folgenden 


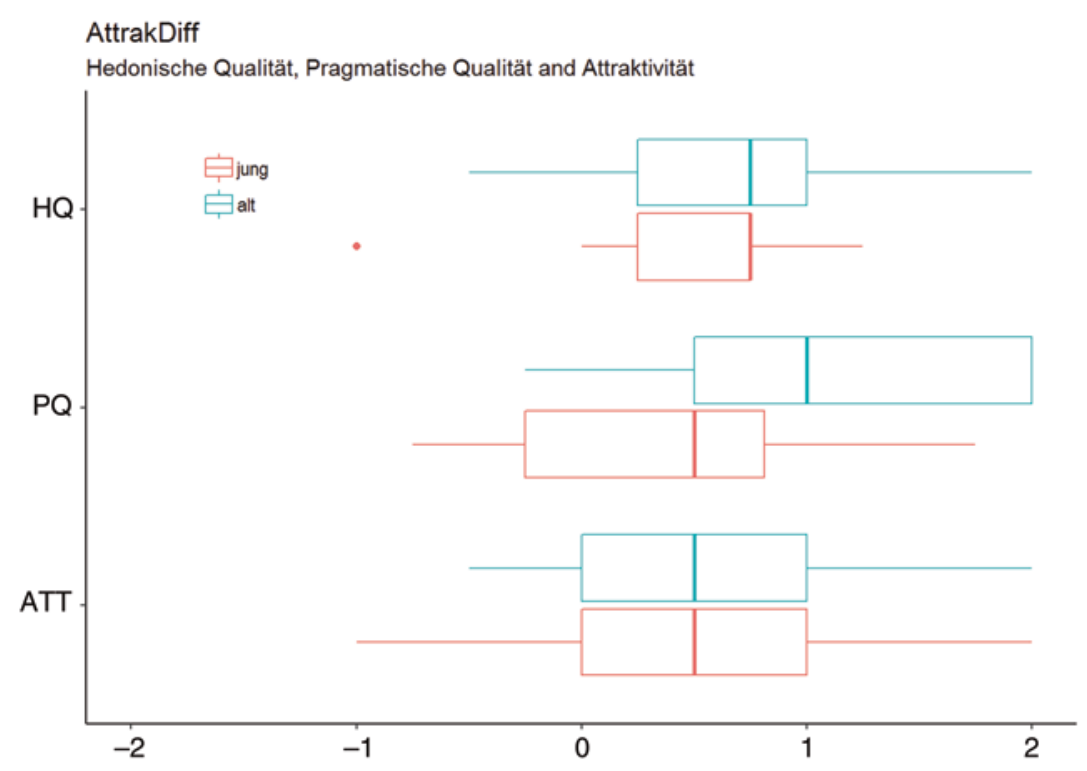

Abb. 7.2 Ergebnisse des AttrakDiff-Fragebogens aufgeschlüsselt nach Alter. Attraktivität (ATT) und hedonische Qualität (HQ) zeigen dabei kaum Unterschiede, während ältere Probanden die pragmatische Qualität (PQ) als signifikant besser bewerteten

werden die erarbeiteten Themenbereiche (Wissen, Erfahrung, Sicherheit und Vertrauen, sowie Kommunikation und Interaktion) mit den jeweiligen Statements vorgestellt.

\section{Kommunikation und Interaktion}

Der Großteil der im Interview gestellten Fragen betraf die Interaktion und Kommunikation mit dem Shuttle sowie automatisierten Fahrzeugen generell.

Unter anderem wurde die Frage gestellt, wie automatisierte Fahrzeuge mögliche Systemfehler nach außen hin kommunizieren sollten. $(n=6)$ Personen erwähnten in diesem Zusammenhang visuelle Warnungen durch Lichtsignale - manche Teilnehmer wiesen explizit auf existierende Konzepte wie Ampelsysteme oder Warnblinkanlagen hin. Ebenso viele $(n=6)$ erwarten auditive Warnungen durch Hupen oder Sprachausgabe. Auch eine Kombination aus beiden Warntypen wurde von $(n=6)$ Personen gewünscht. Auch sollte ein automatisiertes Fahrzeug einem VRU kommunizieren, dass er/sie gesehen wurde. $(n=8)$ Personen erwarten sich dabei multi-modale Nachrichten, welche VRUs sowohl visuell als auch auditiv benachrichtigen. $(n=6)$ Probanden kämen auch mit nur visuellen, $(n=3)$ mit rein auditiven Benachrichtigungen aus.

Bei der Frage, ob der Status von automatisierten Fahrzeugen sowie der Verkehrssituation im Allgemeinen auf digitalen Assistenten (wie Smartphones oder Smartwatches) wiedergegeben werden sollte, hat sich die Meinung der Stichprobe polarisiert. $(n=15)$ Personen konnten sich gut vorstellen, derartige Informationen (z. B. die Position die Route des Fahrzeuges, Anschlussverbindungen, etc.) auf mobilen Endgeräten zu erhalten. Gleich 
viele $(n=15)$ Teilnehmer gaben jedoch auch an, derartige Systeme abzulehnen. Die Hauptgründe dafür waren, dass nicht jeder ein solches Endgerät besitzt beziehungsweise benutzen möchte. Auch Datenschutzbedenken spielen eine Rolle - einige Teilnehmer lehnen es ab, Mobilitätsanbietern ihre privaten Daten zur Verfügung zu stellen.

Auf die Frage, wie man automatisierte Fahrzeuge sicherer gestalten könnte, gaben nur wenige Teilnehmer konkrete Antworten. $(n=5)$ Teilnehmer merkten an, dass zu jeder Zeit eine verantwortliche Person (wie der Operator des Shuttles) innerhalb des Fahrzeugs erreichbar sein sollte, $(n=4)$ gaben an, dass ein für jede(n) zugänglicher Notaus-Schalter angebracht sein sollte.

Es wurde auch explizit nach potenziell problematischen Situationen gefragt, welche bei der Interaktion mit automatisierten Fahrzeugen auftreten könnten. Die dabei erhaltenen Antworten lassen sich grob in drei unterschiedliche Problemsituationen einteilen: (1) keine oder missverständliche Kommunikation, welche von VRUs nicht eindeutig interpretiert werden kann, (2) dass VRUs von Systemen erst gar nicht erkannt werden, und (3) dass Kommunikationssysteme, wenn vorhanden, Probleme mit beeinträchtigten (wie etwa blinden oder tauben Personen) VRUs haben könnten.

Auf die Frage, ob das in dieser Studie betrachtete automatisierte Shuttle genug Informationen für VRUs bereitstellt, gaben $(n=17)$ Personen an, ausreichend über das Verhalten Bescheid zu wissen, jedoch in erster Linie durch Wissen über die Route bzw. durch Beobachten des Verhaltens. $(n=8)$ Teilnehmer gaben an, nicht ausreichend informiert zu sein.

Da das Shuttle zum Untersuchungszeitpunkt mit relativ geringer Geschwindigkeit unterwegs war, wurde auch die Frage gestellt, ob und wie sich der Kommunikationsbedarf ändern würde, wenn höhere Geschwindigkeiten erreicht würden. Für ( $n=11)$ Teilnehmer würde es ausreichen, wenn sich derartige Fahrzeuge an die Verkehrsregeln halten bzw. ein mit menschlichen Verkehrsteilnehmern vergleichbares Verhalten an den Tag legen. Weitere $(n=11)$ Personen betonten, dass automatisierte Fahrzeuge nur dann schneller fahren sollten, wenn die Technologie auch ausgereift genug ist, um vorausschauend zu fahren, angemessen schnell zu reagieren, und dabei auch in der Lage ist, mit anderen Verkehrsteilnehmern zu kommunizieren. $(n=4)$ Teilnehmer betonten, dass zukünftige Kommunikationssysteme einem leicht zu erlernenden und zu definierenden Standard entsprechen müssen.

\section{Sicherheit und Vertrauen}

Ein großer Teil der Probanden $(n=19)$ gaben an, dass sie automatisierte Fahrzeuge für sicher hielten. Diese hatten ein hohes Vertrauen in Technologie und bescheinigten selbiger, sicherer und weniger fehleranfällig zu sein als Menschen - vor allem unter passenden Bedingungen (,bei geringer Geschwindigkeit und guten Wetter- und Sichtverhältnissen“). Vorausgesetzt wird dabei, dass diese Fahrzeuge sich an die Straßenverkehrsordnung halten und rigorose Zulassungsprozesse durchlaufen müssen, denen in Deutschland üblicherweise vertraut wird. $(n=11)$ Teilnehmer gaben andererseits jedoch an, automatisierte Fahrzeuge für nicht besonders sicher zu halten. Gründe hierfür waren aktuelle Medienberichte über Unfälle mit automatisierten Fahrzeugen, Befürchtungen, dass die Technologie 
noch zu wenig ausgereift ist, oder dass es im Mischverkehr mit manuell gesteuerten Fahrzeugen zu Problemen kommen könnte. Einige gaben auch explizit an, dass das Fehlen von Kommunikationsmöglichkeiten zu sicherheitskritischen Situationen führen wird. Trotzdem gaben auch fast zwei Drittel $(n=20)$ an, den Herstellern bei der Entwicklung der Fahrzeuge zu vertrauen. Insbesondere deutsche Hersteller wurden von den Teilnehmern hervorgehoben, mit dem Hinweis, dass diese auch in der Vergangenheit besonders in Sicherheitssysteme investiert haben.

\section{Wissen}

Fast die Hälfte der Versuchsteilnehmer $(n=15,47 \%)$ gab an, bereits einiges über automatisierte Fahrzeuge und deren Funktionsweise zu wissen. Die dabei am häufigsten genannten Begriffe waren „Sensorik“, „Kameras“, und „Programmieren“. Sechs Probanden gaben auch an, ein Basisverständnis von künstlicher Intelligenz zu besitzen, und stützten ihre Angaben mit Begriffen wie ,digitale Sprachverarbeitung“ oder ,lernfähige neuronale Netze“. Nur vier Teilnehmer gaben an, noch kaum etwas über automatisiertes Fahren zu wissen. Der Großteil ( $n=23)$ gab an, ihr Wissen über automatisierte Fahrzeuge vor allem über Printmedien oder Fernsehbeiträge erlangt zu haben, fünf weitere in erster Linie über Mundpropaganda, z. B. durch Familienmitglieder.

\section{Erfahrung}

Die Versuchsteilnehmer wurden auch angehalten, existierende Erfahrungen im Umgang mit dem automatisierten Shuttle anhand konkreter Situationen zu beschreiben. $(n=10)$ Teilnehmer gaben an, dass das Shuttle ohne Probleme funktioniert. Einige gaben jedoch auch an, dass das Fahrzeug das Verhalten nicht klar genug signalisiert. $(n=13)$ Probanden sind auch schon als Passagiere mit dem Shuttle gefahren, wobei $(n=10)$ ihre diesbezüglichen Erfahrungen als angenehm bezeichneten. $(n=4)$ würden eine höhere Geschwindigkeit bevorzugen, $(n=2)$ Teilnehmer gaben explizit an, dass das Shuttle zu langsam fährt, während eine weitere Person überhaupt manuelles Fahren bevorzugte.

\subsubsection{Videoanalyse}

Zusätzlich zu den Interviews wurde an den beiden Tagen der Studie eine GoPro-Kamera am Shuttle angebracht, welche das Geschehen direkt in Sichtlinie des Fahrzeuges aufnahm (siehe Abb. 7.3). Durch den relativ engen Bildausschnitt war es zwar nicht möglich, das Geschehen an den Längs- und der Rückseite des Shuttles zu erfassen, trotzdem konnten einige interessante Aspekte identifiziert werden. Das Videomaterial (mit einer Gesamtdauer von mehr als fünf Stunden) wurde per Hand annotiert - dabei wurden sämtliche Situationen erfasst, welche Interaktionen des Fahrzeugs mit anderen Verkehrsteilnehmern beschrieben. Die Analyse bietet einen guten Einblick in die Schwächen der aktuell eingesetzten Technologie.

\section{Probleme mit VRUs im Längsverkehr}

Insgesamt wurden $(n=30)$ Situationen identifiziert, in denen ungeschützte Verkehrsteilnehmer (zumeist Fußgänger) dem Shuttle entgegen kamen und seitlich (links oder rechts) 

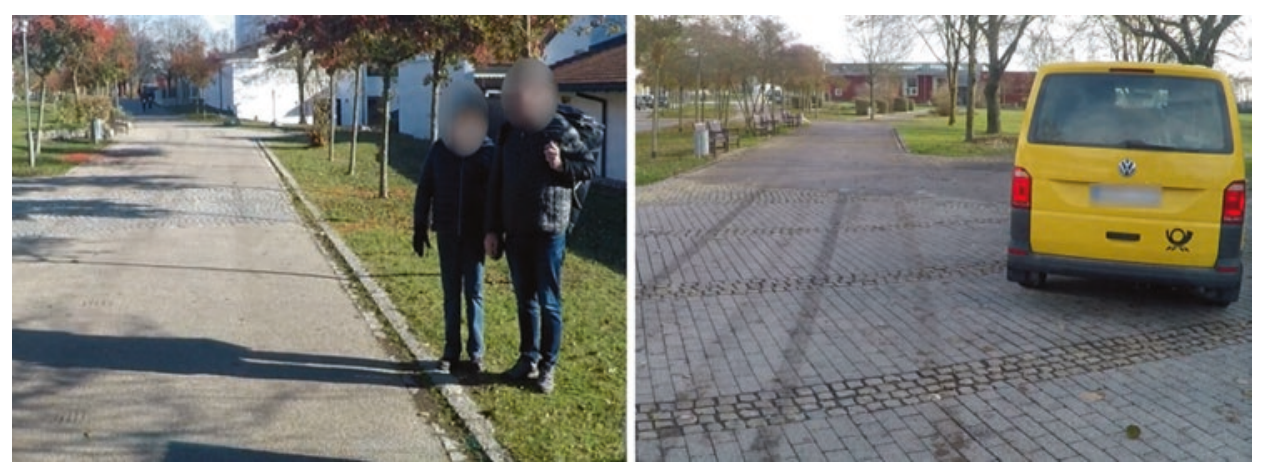

Abb. 7.3 Typische wiederkehrende Situationen aus der Ego-Perspektive des Shuttles: Obwohl entgegenkommende Personen ausweichen, bremst das Shuttle (links), ein stehendes Fahrzeug verhindert die Weiterfahrt (rechts)

an diesem vorbeigingen, wobei das Shuttle aktiv einen Bremsvorgang einleitete. In vielen dieser Situationen war der Seitenabstand sogar größer als typischerweise beim Zusammentreffen mit manuell gesteuerten Fahrzeugen. Auch wichen VRUs manchmal sogar auf die Wiese oder das Bankett aus und waren darüber verwundert, dass das Shuttle trotzdem die Geschwindigkeit verringerte. Laut technischer Spezifikation verringert das Shuttle die Geschwindigkeit, wenn sich ein Objekt im Umkreis von 1,5 Metern befindet (siehe Kap. 5). Für VRUs ist es somit äußerst schwierig zu erkennen, welchen Abstand sie zum Fahrzeug halten müssen, um den Betrieb nicht zu beeinflussen. In weiteren $(n=6)$ Situationen gingen Fußgänger direkt vor dem Shuttle und zwangen dieses zu einem Bremsvorgang. In manchen dieser Situationen musste das Shuttle dabei aktiv ein akustisches Signal aussenden, um sein Kommen anzukündigen, in anderen hatte man als Beobachter sogar den Eindruck, dass bestimmte Personen von der Präsenz des Shuttles wussten, aber trotzdem ihre Pfade nicht anpassten.

\section{Probleme beim Zusammentreffen mit anderen Fahrzeugen}

In insgesamt $(n=7)$ Situationen musste das Shuttle wegen Fahrzeugen auf der Strecke vollständig anhalten. Um die Situation zu bereinigen, musste der Operator des Shuttles daraufhin die Hindernisse manuell umfahren oder warten, bis die Strecke wieder frei war. Einer der Operatoren vermutete, auf derartige Situationen angesprochen, dass manche (andere Verkehrsteilnehmer) dies sogar absichtlich machen würden, um den Betrieb zu beeinträchtigen. In $(n=12)$ Situationen mit Gegenverkehr bremste das Shuttle oder hielt vollständig an, obwohl genügend Platz zum Ausweichen vorhanden gewesen wäre. Das Shuttle selbst ist mit der aktuellen Programmierung nicht in der Lage, seine Route zu verlassen, um den vorhandenen Platz auszunutzen - was für die Fahrer der entgegenkommenden Fahrzeuge jedoch nicht selbstverständlich schien.

\section{Probleme im Querverkehr}

Durch die spezielle Topologie der Route konnten nur wenige (nicht-geregelte) Konfliktsituationen identifiziert werden, in denen VRUs vor dem Shuttle die Straße überquerten. 
In $(n=3)$ Fällen überquerten Fußgänger relativ knapp (<10 Meter) die Straße, ohne das Shuttle zu beachten. Zwei Situationen wurden aufgezeichnet, in denen Fußgänger zuerst zögerten, das Überqueren jedoch einleiteten, als sie erkannten, dass das Shuttle die Geschwindigkeit verringert. Während des Überquerens wurde das Shuttle dabei genau im Auge behalten.

Überhaupt wurden zahlreiche Situationen erfasst, in welchen Fußgänger (querend oder entgegenkommend) ihre Aufmerksamkeit stark auf das Shuttle richteten. Aus den Videoaufzeichnungen kann jedoch nicht entnommen werden, ob dies aus allgemeinem Interesse an automatisierten Fahrzeugen geschah, oder ob die Fußgänger dies aus Sicherheitsgründen für notwendig hielten.

\subsection{Diskussion}

Die Ergebnisse der Feldstudie liefern einige interessante Erkenntnisse in Hinblick auf die gestellten Forschungsfragen. Bezüglich der evaluierten Fragebögen lässt sich feststellen, dass die allgemeine Akzeptanz gegenüber automatisierten Fahrzeugen hoch ist, und die von Reig et al. (2018) gewonnenen Ergebnisse bestätigt werden können. Aus der Sicht von VRUs zeigt sich zusätzlich ein Zusammenhang zur allgemeinen Einstellung gegenüber künstlicher Intelligenz und neuartigen Technologien im Allgemeinen. Personen, welche diesbezüglich aufgeschlossen sind, scheinen automatisierten Fahrzeugen gegenüber offen und glauben auch, dass diese einen Sicherheitsgewinn darstellen. Dies ist insbesondere wertvoll, da es sich bei dieser Studie nicht nur um eine andere Stichprobe unter anderen kulturellen Voraussetzungen, sondern auch um ein anderes Fahrzeug handelt. Des Weiteren legen die Ergebnisse nahe, dass zumindest aus der Sicht von VRUs keine großen Unterschiede zwischen jüngeren und älteren Personen erkennbar sind. Ältere Personen haben das Shuttle bezüglich Akzeptanz und pragmatischer Qualität zwar signifikant besser bewertet, allerdings könnte dieser Unterschied auch durch Interviewer-Bias entstanden sein - möglicherweise wollten sich ältere Probanden gegenüber den Experimentleitern als besonders aufgeschlossen zeigen. Auch was die Erwartungen an die Kommunikationssysteme automatisierter Fahrzeuge betrifft, waren in der Inhaltsanalyse der Interviews kaum Unterschiede zwischen den beiden Zielgruppen zu erkennen. Im Gegensatz zu den bisher präsentierten und technisch ausgefallenen Lösungen, scheinen VRUs in erster Linie Konzepte zu bevorzugen, die bereits aus dem Straßenverkehr bekannt sind, wie Ampelsysteme oder Klingeln/Hupen. Wichtig erscheint vor allem der Wunsch nach unmissverständlich klaren und standardisierten Systemen $(R Q 1)$.

Die Ergebnisse der durchgeführten Videoanalyse, bei der potenziell missverständliche Situationen beim Zusammentreffen von VRUs mit automatisierten Fahrzeugen identifiziert werden sollten, decken sich mit den subjektiv erhobenen Daten. Vorhersehbares Verhalten von automatisierten Fahrzeugen erscheint besonders wichtig zu sein, um VRUs zu ermöglichen, sich selbst an die Situation anzupassen. Ein Szenario, welches dabei oft betrachtet wird, kam in der Videoanalyse selten vor: Kommunikation beim Überqueren der Straße. Ähnlich wie bei Rothenbücher et al. (2016) scheinen VRUs nicht häufig über den 
Vorrang zu verhandeln, sondern die Straße dann zu überqueren, wenn das Fahrzeug noch weit genug entfernt ist bzw. die Geschwindigkeit verringert. Dafür trat in der Videoanalyse eine ganz andere Situation in großer Häufigkeit in den Vordergrund: Kommen dem automatisierten Shuttle VRUs oder manuell gesteuerte Fahrzeuge entgegen, so führt im manuellen Verkehr gelerntes Verhalten schnell zu Problemen. Erstens bewegt sich das Shuttle auf einer festen Bahn zwischen den Spurmarkierungen und weicht auch dann nicht aus, wenn genügend Platz vorhanden wäre. Zusätzlich ist es für VRUs kaum erkennbar, welche Abstände sie einhalten müssen, um den Betrieb des Shuttles nicht zu beeinträchtigen. Studienteilnehmer wiesen in Interviews darauf hin, dass das Shuttle kommunizieren sollte, ob ein VRU erfasst wurde, und waren oft verwundert, warum das Shuttle die Geschwindigkeit verringert, obwohl sie doch einen an sich ungefährlichen und großen Seitenabstand einhielten (RQ2). Dies zeigt, dass automatisierte Fahrzeuge anderen Verkehrsteilnehmern kommunizieren sollten, wenn diese dem Fahrzeug zu nahe kommen - hierfür könnte man zum Beispiel den einzuhaltenden Sicherheitsabstand rund um das Fahrzeug auf den Boden projizieren oder ein Eindringen in diesen mit LED-Streifen/Lichtsignalen sichtbar machen. Im Hinblick darauf, dass moderne Verkehrsplanung oft die Wichtigkeit von sogenannten „Shared Spaces“ (Hamilton-Baillie 2004) betont, in welchen sämtliche Verkehrsteilnehmer gleichwertig behandelt werden, müssen derartige Konflikte vermieden werden.

\subsubsection{Einschränkungen und zukünftige Arbeiten}

Zukünftige Studien müssen die Größe und Diversität der Stichprobe erhöhen, um die erhobenen Erkenntnisse zu generalisieren. Auch die niedrige Geschwindigkeit des Shuttles, sowie die - im Vergleich zu größeren und dichter besiedelten Städten - niedrige Einwohnerzahl in Bad Birnbach könnten die Studie beeinflusst haben. Man könnte allerdings auch vermuten, dass die Anzahl der hier demonstrierten Problemsituationen in dichter besiedelten urbanen Regionen weiter steigt. Durch den engen Bildausschnitt der verwendeten GoPro-Kamera lässt sich des Weiteren nicht ausschließen, dass andere wichtige Situationen erst gar nicht erfasst wurden. Nichtsdestotrotz können aus den gewonnenen Erkenntnissen einige Empfehlungen für zukünftige Arbeiten abgegeben werden:

\subsubsection{Implikationen für AV/VRU-Interaktionsdesign}

Ungeschützten Verkehrsteilnehmern sollte ermöglicht werden, zukünftige Aktionen von automatisierten Fahrzeugen vorherzusagen. Diese erwarten sich ein ähnliches Verhalten wie auch bei manuell gesteuerten Fahrzeugen. Automatisierte Fahrzeuge sollen sich an die Verkehrsregeln halten und die Geschwindigkeit verringern, wenn ein VRU die Straße überqueren möchte. Sollte Kommunikation doch notwendig sein, reichen einfache Hinweise (Lichtsignale, Hupen, etc.), welche bekannten Konzepten wie Ampelsystemen oder Verkehrsschildern ähneln sollen. Dabei ist es ebenfalls wichtig, auch Personen mit Einschränkungen (z. B. Personen mit Seh- oder Gehörschwäche) zu berücksichtigen. Auch wünschen sich VRUs ein ähnliches Verhalten von allen Fahrzeugen, was die Dringlichkeit von Standards für Kommunikationssysteme hervorhebt. 


\subsubsection{Implikationen für zukünftige Arbeiten}

Ein automatisiertes Fahrzeug sollte VRUs explizit kommunizieren, dass diese von den Sensorsystemen erkannt wurden. Während das klassische Überqueren der Straße vor dem Fahrzeug nicht häufig zu Unsicherheiten führte, traten Probleme vor allem dann auf, wenn VRUs dem Fahrzeug entgegen kamen oder sich neben diesem befanden. Speziell in Shared Spaces, in welchen häufige Interaktionen zwischen VRUs und automatisierten Fahrzeugen zu erwarten sind, muss klar sein, wo der Bereich beginnt/endet, in dem das Fahrzeug auf andere Verkehrsteilnehmer reagiert.

\subsection{Fazit}

In diesem Kapitel wurde die Akzeptanz von automatisierten Fahrzeugen aus Sicht ungeschützter Verkehrsteilnehmer evaluiert. Dazu wurden (1) potenziell problematische Situationen identifiziert, welche sich durch Interaktion im Außenraum ergeben sowie (2) Anforderungen an zukünftige Kommunikationssysteme erhoben. Die Resultate zeigen, dass die Akzeptanz gegenüber automatisierten Fahrzeugen hoch ist und stark von der Einstellung gegenüber technischen Neuerungen allgemein abhängt. Zusätzlich wurde gezeigt, dass häufig genannte Problemsituationen (wie explizite Kommunikation beim Überqueren der Straße) in der Realität in nur geringer Häufigkeit vorkamen, während andere Situationen regelmäßig zu Problemen führten. In besonders vielen Situationen reagierte das Fahrzeug aktiv auf Personen, welche eigentlich versuchten, den Einflussbereich des Shuttles zu verlassen. Für einen reibungslosen Betrieb sollen zukünftige Fahrzeuge ihre Absichten und ihren Systemzustand kommunizieren, und dabei die im Rahmen der Studie erhobenen Benutzeranforderungen erfüllen. Ungeschützte Verkehrsteilnehmer bevorzugen dabei einfache und schnell interpretierbare Kommunikationsmethoden, welche standardisiert sind und anderen aus dem Verkehr bekannten Konzepten ähnlich sind. Weitere Details zu dieser Studie finden Sie in Löcken et al. (2019).

\subsection{Danksagung}

Wir bedanken uns bei der Gemeinde Bad Birnbach, der Geschäftsführung der Rottal Terme, sowie Isabella Thang für die Unterstützung bei der Rekrutierung der Versuchsteilnehmer sowie der Durchführung der Studie.

\section{Anhänge}

\section{Anhang 1 - Fragebogen}

Für folgende Statements mussten Studienteilnehmer auf einer 5-Punkte-Likert-Skala angeben, inwieweit sie den jeweiligen Aussagen zustimmen $(0=$ Stimme überhaupt nicht zu; $5=$ Stimme voll und ganz zu): 
1. AAV Automatisiertes Fahren ist wichtig für mich.

2. AAV Automatisiertes Fahren ist wichtig für die Gesellschaft.

3. AAV Automatisierte Fahrzeuge sind vertrauenswürdig.

4. AAV Ich bin an automatisierten Fahrzeugen interessiert.

5. AKI Künstliche Intelligenz ist wichtig für mich.

6. AKI Künstliche Intelligenz ist wichtig für die Gesellschaft.

7. AKI Künstliche Intelligenz ist vertrauenswürdig.

8. AKI Ich bin an künstlicher Intelligenz interessiert.

9. AT Neue Technologien (wie Smartphones, Virtual Reality, etc.) sind wichtig für mich.

10. AT Neue Technologien (wie Smartphones, Virtual Reality, etc.) sind wichtig für die Gesellschaft.

11. AT Ich vertraue neuen Technologien (wie Smartphones, Virtual Reality, etc.).

12. AT Ich bin an neuen Technologien (wie Smartphones, Virtual Reality, etc.) interessiert.

13. PRED Ich hatte zu jeder Zeit das Gefühl, darüber informiert zu sein, was das Fahrzeug als nächstes tun wird.

14. PRED Ich konnte das Verhalten des automatisierten Fahrzeuges vorhersagen.

15. BEHAVIOR Ich verhalte mich anders, wenn ich einem automatisierten Fahrzeug begegne (im Vergleich zu einem manuell gesteuerten Fahrzeug).

16. MENTAL Ich fühle mich bei Begegnungen mit dem automatisierten Fahrzeug überfordert.

17. RISK Ich halte auch am Fußgängerüberweg an, da ich nicht weiß, ob das automatisierte Fahrzeug stehen bleibt.

18. PLAY Ich würde gerne austesten, ob das Fahrzeug bremst, wenn ich mich in den Weg stelle.

19. COMM Wenn kein Mensch am Steuer ist, ist es schwieriger, zu kommunizieren (z. B. Vorfahrt verhandeln).

\section{Anhang 2 - Interviewfragen}

Folgende Fragen wurden von den Studienteilnehmern in Form semi-strukturierter Interviews beantwortet und durch Inhaltsanalyse ausgewertet. Dabei wurden zusammenpassende Statements, unabhängig von der jeweilig gestellten Frage, zu verschiedenen Themengebieten aggregiert.

1. Beschreiben Sie die gerade erlebte Verkehrssituation mit dem automatisierten Shuttle. Hat es sich fehlerfrei verhalten? Haben andere Verkehrsteilnehmer (Fahrzeuge, Fußgänger) darauf reagiert? Haben andere Verkehrsteilnehmer den Eindruck erweckt, dem Shuttle zu vertrauen?

2. Glauben Sie, dass automatisierte Fahrzeuge sicher sind? Warum/warum nicht?

3. Haben Sie bereits viel über automatisierte Fahrzeuge gehört? Wenn ja, aus welchen Quellen? 
4. Vertrauen Sie der Vorgehensweise der Hersteller bei der Entwicklung von automatisierten Fahrzeugen?

5. Haben Sie Angst, dass automatisierte Fahrzeuge versagen könnten? Falls ja, nennen sie bitte ein bis drei Szenarien, in welchen Sie glauben, dass automatisierte Fahrzeuge versagen könnten.

6. Wenn ein Fehler passiert, wie könnte ein automatisiertes Fahrzeug dies einem Fußgänger, Radfahrer, oder Fahrer eines Fahrzeuges klar machen?

7. Was könnte ein automatisiertes Fahrzeug machen, damit Sie weniger Angst vor Fehlern haben?

8. Benützen Sie regelmäßig einen persönlichen Assistenten mit künstlicher Intelligenz, wie etwa Apple Siri, Amazon Alexa oder Google Now? Wenn ja, für welche Zwecke?

9. Würden Sie es begrüßen, wenn Ihnen derartige Assistenten, Smartphones oder Smartwatches Informationen über automatisierte Fahrzeuge in der Nähe geben würden?

10. Fahren Sie regelmäßig selbst mit einem Fahrzeug?

11. Sind Sie schon einmal mit einem automatisierten Fahrzeug mitgefahren? Wenn ja, beschreiben Sie bitte kurz Ihre Erfahrungen.

12. Was wissen Sie über die Funktionsweise von automatisierten Fahrzeugen?

13. Was wissen Sie über die Funktionsweise von künstlicher Intelligenz?

14. Hatten Sie zu jeder Zeit das Gefühl, ausreichend darüber informiert zu sein, was das Fahrzeug als nächstes tun wird (z. B. ob es für andere Verkehrsteilnehmer anhält, etc.)?

15. Können Sie ein Szenario beschreiben, von welchem Sie glauben, dass ein automatisiertes Fahrzeug überfordert wäre?

16. Wie müsste ein automatisiertes Fahrzeug sich verhalten, damit Sie sicher sein können, dass Sie gesehen wurden, bzw. es auf Sie wartet?

17. Können Sie ein oder mehrere Beispiele für schlechte Kommunikation zwischen einem automatisierten Fahrzeug und anderen Verkehrsteilnehmern (Fahrer manueller Fahrzeuge, Fußgänger, Radfahrer, etc.) angeben, oder sich eines ausdenken?

18. Wie müsste sich das automatisierte Fahrzeug verhalten, wenn es mit höherer Geschwindigkeit fahren würde (z. B. ähnliche Geschwindigkeit wie ,normale“ Fahrzeuge)?

\section{Literatur}

Böckle, M. P., Brenden, A. P., Klingegard, M., Habibovic, A., Bout, M. (2017, September). SAV2P: Exploring the Impact of an Interface for Shared Automated Vehicles on Pedestrians' Experience. In Proceedings of the 9th International Conference on Automotive User Interfaces and Interactive Vehicular Applications Adjunct (pp. 136-140). ACM.

Courage, C., Baxter, K., Understanding your users: A practical guide to user requirements methods, tools, and techniques. GulfProfessional Publishing, 2005.

Dey, D., Terken, J. (2017, September). Pedestrian interaction with vehicles: roles of explicit and implicit communication. In Proceedings of the 9th International Conference on Automotive User Interfaces and Interactive Vehicular Applications (pp. 109-113). ACM. 
Dey, D., Martens, M., Wang, C., Ros, F., Terken, J. (2018, September). Interface Concepts for Intent Communication from Autonomous Vehicles to Vulnerable Road Users. In Adjunct Proceedings of the 10th International Conference on Automotive User Interfaces and Interactive Vehicular Applications (pp. 82-86). ACM.

Färber, B. (2015). Kommunikationsprobleme zwischen autonomen Fahrzeugen und menschlichen Fahrern. In Autonomes Fahren (pp. 127-146). Springer Vieweg, Berlin, Heidelberg.

Hamilton-Baillie, B., „Urban design: Why don't we do it in the road? modifying traffic behavior through legible urban design," Journal of Urban Technology, vol. 11, no. 1, pp. 43-62, 2004.

Hassenzahl, M., Burmester, M., Koller, F. (2003). AttrakDiff: Ein Fragebogen zur Messung wahrgenommener hedonischer und pragmatischer Qualität. In Mensch und Computer 2003 (pp. 187196). Vieweg + Teubner Verlag.

Jain, A., Gupta, A., Rastogi, R. (2014). Pedestrian crossing behaviour analysis at intersections. International Journal for Traffic and Transport Engineering, 4(1), 103-116.

Jiang, X., Wang, W., Bengler, K., Guo, W. (2015). Analyses of pedestrian behavior on mid-block unsignalized crosswalk comparing Chinese and German cases. Advances in mechanical engineering, 7(11), 1687814015610468.

Lagstrom, T., Lundgren, V. M. (2015). AVIP-Autonomous vehicles interaction with pedestrians. Master of Science Thesis, Chalmers University of Technology.

Lehsing, C., Benz, T., Bengler, K. (2016). Insights into interaction-effects of human-human interaction in pedestrian crossing situations using a linked simulator environment. IFAC-PapersOnLine, 49(19), 138-143.

Löcken, A., Wintersberger, P., Frison, A., Riener, A. (2019). Investigating user requirements for communication between automated vehicles and vulnerable road users. 2019 IEEE Intelligent Vehicles Symposium (IV), Paris, France, pp. 879-884.

Lundgren, V. M., Habibovic, A., Andersson, J., Lagström, T., Nilsson, M., Sirkka, A., ... Saluäär, D. (2017). Will there be new communication needs when introducing automated vehicles to the urban context?. In Advances in Human Aspects of Transportation (pp. 485-497). Springer, Cham.

Mahadevan, K., Somanath, S., Sharlin, E. (2018, April). Communicating Awareness and Intent in Autonomous Vehicle-Pedestrian Interaction. In Proceedings of the $2018 \mathrm{CHI}$ Conference on Human Factors in Computing Systems (p. 429). ACM.

Mirnig, A. G., Wintersberger, P., Meschtscherjakov, A., Riener, A., Boll, S. (2018, September). Workshop on Communication between Automated Vehicles and Vulnerable Road Users. In Adjunct Proceedings of the 10th International Conference on Automotive User Interfaces and Interactive Vehicular Applications (pp. 65-71). ACM.

OECD/ITF. 2014. Road Safety Annual Report. Technical Report. OECD Publishing.

Pennycooke, N. (2012). AEVITA: designing biomimetic vehicle-to-pedestrian communication protocols for autonomously operating and parking on-road electric vehicles (Doctoral dissertation, Massachusetts Institute of Technology).

Reig, S., Norman, S., Morales, C. G., Das, S., Steinfeld, A., Forlizzi, J. (2018, September). A Field Study of Pedestrians and Autonomous Vehicles. In Proceedings of the 10th International Conference on Automotive User Interfaces and Interactive Vehicular Applications (pp. 198-209). ACM.

Rothenbücher, D., Li, J., Sirkin, D., Mok, B., Ju, W. (2016). Ghost driver: A field study investigating the interaction between pedestrians and driverless vehicles. 2016 25th IEEE International Symposium on Robot and Human Interactive Communication (RO-MAN), New York, NY, pp. 795-802.

Sucha, M. (2014). Road users' strategies and communication: driver-pedestrian interaction. Transport Research Arena (TRA).

Sucha, M., Dostal, D., Risser, R. (2017). Pedestrian-driver communication and decision strategies at marked crossings. Accident Analysis Prevention, 102, 41-50. 
Open Access Dieses Kapitel wird unter der Creative Commons Namensnennung 4.0 International Lizenz (http://creativecommons.org/licenses/by/4.0/deed.de) veröffentlicht, welche die Nutzung, Vervielfältigung, Bearbeitung, Verbreitung und Wiedergabe in jeglichem Medium und Format erlaubt, sofern Sie den/die ursprünglichen Autor(en) und die Quelle ordnungsgemäß nennen, einen Link zur Creative Commons Lizenz beifügen und angeben, ob Änderungen vorgenommen wurden.

Die in diesem Kapitel enthaltenen Bilder und sonstiges Drittmaterial unterliegen ebenfalls der genannten Creative Commons Lizenz, sofern sich aus der Abbildungslegende nichts anderes ergibt. Sofern das betreffende Material nicht unter der genannten Creative Commons Lizenz steht und die betreffende Handlung nicht nach gesetzlichen Vorschriften erlaubt ist, ist für die oben aufgeführten Weiterverwendungen des Materials die Einwilligung des jeweiligen Rechteinhabers einzuholen.

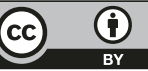

